\title{
Stability Analysis of an Expansive Clay Slope: A Case Study of Infiltration- Induced Shallow Failure of an Embankment in Regina, Canada
}

\author{
Shunchao Qi and Sai K. Vanapalli \\ Department of Civil Engineering, University of Ottawa \\ Ottawa, ON, K1N 6N5, Canada
}

\begin{abstract}
An expansive clay slope in Regina, Canada that failed in the spring of 1973 is reanalyzed using a comprehensive numerical procedure that involves the coupled hydro-mechanical finite element analysis. This procedure reliably simulates the behavior of the slope for computing its factor of safety (FS) under snow melting infiltration condition. The results of the study show that a bimodal permeability function with an increased saturated coefficient of permeability by 2-3 of magnitudes in comparison to the laboratory measured value can be used to reasonably describe the hydraulic properties of unsaturated cracked expansive soils and the effect of cracks on slope stability. An increase in saturated coefficient of permeability (up to the rate of snow melting) and use of residual shear strength parameters contribute to early failure of this slope under snow melting condition. The study highlights the use of field saturated coefficient of permeability and bimodal permeability function such that expansive soil slope stability can be reliably estimated.
\end{abstract}

Keywords: expansive soils, shallow slope failure, snow melting infiltration, cracks, coupled hydromechanical model, bimodal permeability function, factor of safety, Regina clay

\section{Introduction}

Infiltration (rain or snow)-induced shallow slope failures in expansive soils is one of the major problems that continues to challenge both the researchers and the practitioners in Canada. For example, numerous shallow failures of cut and fill slopes were continuously reported in the city of Regina which is situated on glacial deposit basin consisting of over-consolidated expansive clays (Widger and Fredlund 1978, Chowdhury 2013).

The problems associated with the instability of expansive soil slope under infiltration condition are also common in many other countries (e.g. United States, China, India, and Spain). The response of expansive soil slopes to climate changes (e.g. rainfall or evaporation, etc.) could be significantly different from that of conventional non-expansive clayey soils. Such a behaviour can be attributed to the presence of highly active clay minerals such as the montmorillonite that contribute to considerable volume change upon wetting and drying in expansive soils. In addition, significant cracks and fissures that typically develop in the surface layers have a considerable influence on the infiltration process. The significant complexity of mechanism associated with the retrogressive landslides in expansive soils has been reported in the literature by many researchers (e.g. Flury et al 1994, Hillel 1998, Ng et al 2003, Zhan et al 2007).

In comparison to in-situ tests ( $\mathrm{Ng}$ et al 2003, Zhan et al 2007) or centrifugal tests (Xu et al 2006, Cheng et al 2011), numerical analysis is a more viable and convenient way to reproduce the response of slopes to different infiltration conditions with respect to both time and cost. Hamdhan and Schweiger (2013) conducted a

*Corresponding Author: S. K. Vanapalli, Email: vanapall@eng.uottawa.ca, Tel: 613-562-5800 ext 6638

Copyright (C 2015 Canamaple Academia Services, http://press.camdemia.ca

DOI: 10.15273/ijge.2015.01.003 
Finite Element Method-Based Analysis to simulate the hydraulic behavior of an $11 \mathrm{~m}$ cut slope in Zaoyang, Hubei, China. The response of this slope subjected to an artificial rainfall infiltration was monitored by $\mathrm{Ng}$ et al (2003) using various instruments. The pore water pressures (PWPs) predicted by Hamdhan and Schweiger (2013) showed a reasonably good match with those measured by $\mathrm{Ng}$ et al (2003), except for some small differences, which were attributed to not taking account of cracks and fissures in the numerical analysis. Based on the PWP distribution obtained from Finite Element Analysis (FEA), the variation of local safety factors were computed following the classical expression for infinite slope as a ratio of available shear strength and shear stress by Alonso et al (2003). These results showed that a perched water table developed over the interface between upper two layers due to the strong transition of water storage between them where the minimum FS were consistently found. The water storage can be explained using the difference in the saturated coefficient of permeability and the permeability functions. Rouainia et al (2009) performed a numerical simulation to predict the response of a London clay slope to both present and possible further climate effects; their results highlighted the importance of reliable assessment of the permeability parameters and local climate conditions. The measured saturated coefficient of permeability determined from laboratory tests was increased to account for macroscopic effects of fissures, sand lenses and other heterogeneous features in field conditions, and achieve a better match between the measured and predicted PWP data.

In this paper, the failure of a Regina clay embankment under infiltration of snow melting condition is numerically reanalysed using Finite Element Method, in which the volume change behaviour of Regina clay upon wetting is considered in the coupled hydro-mechanical framework. The effect of fissures and cracks is considered through use of the bimodal (Soil Water Characteristic Curve (SWCC) and bimodal permeability function with an increased saturated coefficient of permeability to represent the in-situ conditions more reliably. The variation of FS with respect to time during the period of snow melting in the spring are presented based on both peak and residual shear strength parameters at the time of failure.

\section{Site Description}

The studied slope is an overpass embankment of the Trans-Canada Highway located at Belle Plaine, Saskatchewan, which is about $45 \mathrm{~km}$ west of Regina (Widger and Fredlund 1978). According to the Köppen Climate Classification System (Ito and Azam 2009), the climate of Regina is classified as semi-arid to arid type. The mean annual precipitation is recorded to be about $370 \mathrm{~mm}$. The temperature at Belle Plaine area varies in a large range from about $-50^{\circ} \mathrm{C}$ in winter to around $43^{\circ} \mathrm{C}$ in summer. The snow depth in the winter can reach $500 \mathrm{~mm}$ in thickness or even higher in this area.

The soil in the studied area is expansive clay, which is widely referred in the literature as Regina clay. The soil properties of Regina clay have been comprehensively investigated by several researchers (e.g. Widger and Fredlund 1978, Shuai 1996, Azam and Ito 2011). Regina clay is a highly plastic soil that contains a significant amount of montmorillonite and exhibits substantial swelling upon wetting and shrinkage during drying. There are several cracks and fissures within the surficial layer of natural Regina clay near the ground surface. In addition, the environmental factors can also lead to a gradual change or degradation in the engineering properties of compacted expansive clay.

During the 1950s and 1960s, several embankments were constructed of Regina clay for the highway overpass at Belle Plaine with two lanes: namely, westbound lane and eastbound lanes (Widger and Fredlund 1978). These embankments had slopes ranging from $4: 1$ to 1.5:1 or even steeper, their maximum fill heights ranged from $9.1 \mathrm{~m}$ to $15.2 \mathrm{~m}$. Several failures were observed in these fills within 4-6 years after their construction, for both steep and relative gentle slope cases. For example, a movement occurred within an embankment, with a slope of 3:1 and height of about $9 \mathrm{~m}$, on northwest face of the eastbound lane in the spring of 1973. This 
slope failure was then visited and investigated by Widger and Fredlund (1978). Fig. 1 shows a cross section along a line with the failed slope surface and measured strength properties. Excavation using a backhoe along this cross section indicated that the slip surface with an essential circular shape was located approximately 2.1 to $2.4 \mathrm{~m}$ below the ground surface. Highly developed fissures were also observed within the surficial layer at the time of failure. A back calculation of FS was carried out by Widger and Fredlund (1978) using the limit equilibrium method along this detected slip surface. This back analysis was mainly based on the conventional saturated soil mechanics, although suction loss was believed to be the primary cause of the failure. This embankment failure is reanalysed in this study extending a comprehensive numerical procedure that utilizes the coupled hydro-mechanical FEA. The influence of stress changes on the FS is also considered.

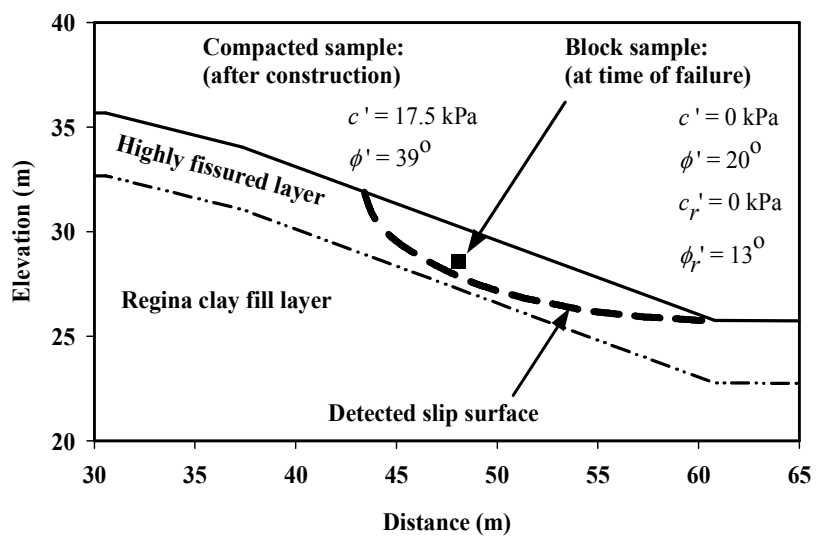

Fig. 1 Cross section of a failed slope (modified after Widger and Fredlund 1978)

\section{Methodology}

\subsection{Response of expansive clay slope to water infiltration}

The response of unsaturated expansive clay to water infiltration is a highly coupled hydromechanical process, including water flow through the unsaturated porous medium and stress change induced by soil swelling. In other words, both the seepage and stress fields within the unsaturated expansive clays keep changing and affecting each other under water infiltration condition. This process should be accounted for and included in appropriate mathematical equations for rational slope stability analysis. The transient flow through the unsaturated porous medium is described by Richards' equation (1931):

$$
\frac{\partial \theta_{w}}{\partial t}=\nabla \cdot\left[k_{\text {unsat }} \nabla\left(\frac{u_{w}}{\gamma_{w} g}+y\right)\right]
$$

where, $\theta_{w}$ is volumetric water content, $t$ is time, $k_{\text {unsat }}$ is the permeability of soils under unsaturated condition, $u_{w}$ is the value of PWP, $\gamma_{w}$ is the density of water, $g$ is the gravitational acceleration, and $y$ is the elevation. Both $\theta_{w}$ and $k_{\text {unsat }}$ are related to $u_{w}$ with nonlinear functions. $\nabla$ is the gradient operator and defined as:

$$
\nabla=\frac{\partial}{\partial x} i+\frac{\partial}{\partial y} j+\frac{\partial}{\partial z} k
$$

The stress field within an unsaturated soil is described using the partial differential equations of static equilibrium as follows:

$$
\nabla(\boldsymbol{\sigma})+\boldsymbol{b}=0
$$

where, $\boldsymbol{\sigma}$ are components of the net total stress tensor, and $\boldsymbol{b}$ are components of the body force vector.

Coupling Eq. (1) and Eq. (2) provides a relatively complete description for the coupled hydro-mechanical response of an unsaturated porous media to water infiltration. With appropriate constitutive relations for unsaturated expansive soils, these two equations can be solved simultaneously to obtain the stress and seepage fields for a specific boundary value problem. In this study, the commercial software SIGMA/W (GeoSlope 2007a) that incorporates the hydraulic analysis code in SEEP/W (GeoSlope 2007b) into static force equilibrium analysis is used for implementing the coupled analysis of the Regina clay slope. The capability of SIGMA/W to conduct coupled saturated and unsaturated hydro-mechanical analysis has been verified by Wong et al (1998) through the Mandel-Cryer problem and a coupled multidimensional consolidation problem for unsaturated soils. 


\subsection{Slope stability evaluation}

The FS value is most commonly used for evaluating the stability of slopes under various environmental conditions. There are many approaches in the literature to solve for FS for one-(e.g. Rahardjo et al 1995, Duncan and Wright 1995), two-(e.g. Morgenstern and Price 1965) and three-(e.g. Lam and Fredlund 1993, Ling et al 2013) dimensional slopes under both saturated and unsaturated conditions. These methods can be generally categorized into three groups: (i) limit equilibrium methods; (ii) finite element stress based methods; and (iii) finite element strength reduction methods. Use of limit equilibrium methods appears to be most popular due to its long history and simplicity (GeoSlope 2007c). The stresses in limit equilibrium methods are typically analysed extending static equilibrium conditions of the failure mass based on some assumptions. They are not always representative of the true stress state conditions in the ground. Comparatively, the stresses from FEA are more realistic and are closer to the actual ground conditions provided that the coefficient of lateral earth pressure at rest $K_{0}$ can be predetermined (GeoSlope 2007c). Therefore, the finite element stress based method seems to be more suitable for the problem in this study, because the stress state within the expansive soils is constantly changing in response to volume change. Based on the calculated stress field using FEA, the FS along a presumed slip surface is defined as the ratio of the resisting and mobilized shear forces integrated along this surface, and has the following equation form:

$$
F S=\frac{\int_{0}^{l} \tau_{f} d l}{\int_{0}^{l} \tau d l}
$$

where, $l$ is the length of presumed slip surface, $\tau$ is the mobilized stress at a point along the slip surface, and $\tau_{f}$ is the soil shear strength at this point.

For soil that is in a state of saturated condition, Mohr-Coulomb failure criterion is well accepted for determining the shear strength for slope stability analysis. Extending the effective stress principle, the Mohr-Coulomb failure criterion is written as:

$$
\tau_{f}=c^{\prime}+\left(\sigma-u_{w}\right) \tan \phi^{\prime}
$$

where, $c^{\prime}$ and $\phi^{\prime}=$ effective cohesion and internal friction angle at saturated condition, $\sigma=$ normal stress acting on the slip surface under the failure mass, and $u_{w}=$ PWP at the corresponding point on the slip surface.

For unsaturated soils, Fredlund and Morgenstern (1976) suggested that two stress state variables, namely, net normal stress and suction, need to be considered independently to adequately describe the mechanical behavior of unsaturated soils. Based on this two stress state variables concept, Vanapalli et al (1996) proposed a semi-empirical model to predict the unsaturated soil shear strength using SWCC as a tool:

$\tau_{f}=c^{\prime}+\left(\sigma-u_{a}\right) \tan \phi^{\prime}+\left(u_{a}-u_{w}\right)\left[\left(\frac{\theta_{w}-\theta_{r}}{\theta_{s}-\theta_{r}}\right) \tan \phi^{\prime}\right]$

where, $\left(\sigma-u_{a}\right)$ is net normal stress, $\left(u_{a}-u_{w}\right)$ is suction, $\theta_{w}$ is volumetric water content, $\theta_{s}$ is saturated volumetric water content, and $\theta_{r}$ is residual volumetric water content. $\theta_{s}$ and $\theta_{r}$ can be obtained from the SWCC using the construction method without using any other fitting parameters. This model was shown to provide reasonable estimates of nonlinear relationship between unsaturated shear strength and suction in the suction range of $0-1,500 \mathrm{kPa}$. Furthermore, a smooth transition can be found between Eqs. (4) and (5). When soil is saturated, $u_{w}$ would be equal to or greater than zero, $u_{a}$ is equal to zero, and $\left(\theta_{w}-\theta_{r}\right) /\left(\theta_{s}-\theta_{r}\right)$ is equal to unity. Eq. (5) numerically reverts to the form valid for saturated conditions embracing the effective stress principle. This semi-empirical model can deal with both the saturated and unsaturated conditions, and therefore is particularly useful to analyze the slip surface 
which is partly above and partly below the ground water table.

The net normal stress, suction or PWP and volumetric water content along a slip surface can be interpolated based on the stress field and seepage field, which are obtained from a coupled hydro-mechanical finite element analysis of the slope profile. Thus, a complete procedure for solving for FS of slope under snow melting condition includes three steps: (i) coupled hydromechanical FEA of slope soil to water infiltration; (ii) computation of the shear strength along a prescribed slip surface using Eq. (5); and (iii) calculation of the overall or global FS using Eq. (3).

\section{Model establishment and soil parameters}

\subsection{Model slope establishment}

As discussed in the previous section, the surficial layer of Regina clay can be significantly affected by environmental factors. The drying and wetting and (or) freezing and thawing cycles can lead to development of fissures and cracks within this layer, which alters the basic structure characteristics of clays, including increase in the void ratio, change in soil fabric, etc. These changes typically affect soil properties and proper values should be used for geotechnical engineering analysis and design. For example, the SWCC and permeability function of cracked soils are shown to be bimodal (Li et al 2011) in nature instead of unimodal as measured on samples compacted in the laboratory. The actual saturated coefficient of permeability at field is typically several magnitudes higher in comparison to that measured in the laboratory. The saturated shear strength parameters will also experience a gradual degradation with the increasing numbers of drying and wetting and (or) freezing and thawing cycles (Pineda et al 2014). Thus, it is more appropriate and reasonable to consider the surficial soils as separate layer when conducting both coupled hydro-mechanical and slope stability analysis. For the case considered in the present study, the top $3 \mathrm{~m}$ are assumed to be the weathered layer with different soil properties resulting mainly from the cracks and fissures as shown in Fig. 2. Aubeny and Lytton (2004) indicated that shallow slip surfaces in several dozen of compacted high plasticity clay slopes ranged from $0.6 \mathrm{~m}$ to $3.0 \mathrm{~m}$. Yoshida et al (1983) estimated the total heave of a slab-on-grade floor on Regina clay considering the active zone depth as $2.8 \mathrm{~m}$. For this reason, the assumption of top 3 $\mathrm{m}$ of weathered layer is considered to be reasonable. Fig. 2 also indicates that the detected slip surface for this case is within the weathered layer. It was reported that this shallow failure occurred in the spring of 1973, when the accumulated snow on the ground was gradually melting. Thus, it is mostly likely that this failure was caused by the water infiltration from snow melting resulting in a decrease in suction and shear strength within the soil. In the meantime, the stress change induced by volume increase upon wetting will have an impact on the slope stability.

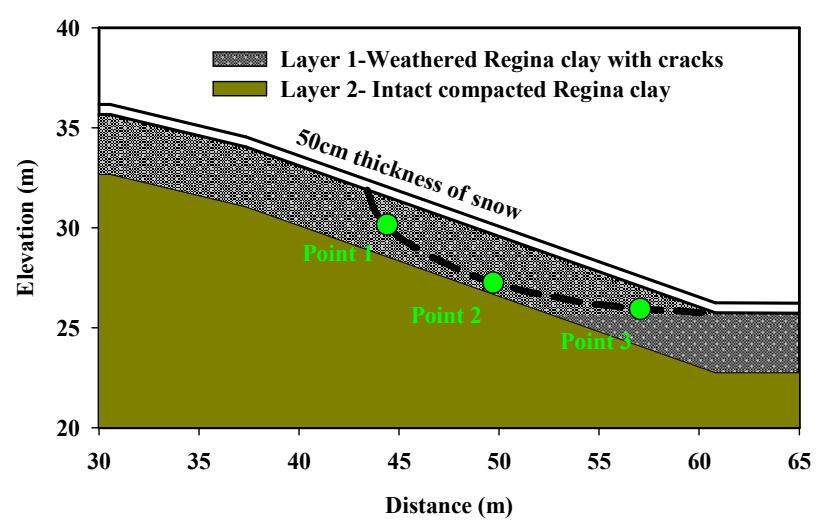

Fig. 2 Conceptual physical model of this slope failure

Based on the conceptual physical model of this slope failure indicated in Fig. 2, a finite element method-based computational model was established as shown in Fig. 3. The initial stress field within this model slope are constructed by running an in-situ analysis, based on the assumption that $K_{0}$ is equal to $\mu /(1-\mu) . K_{0}$ is the coefficient of lateral earth pressure at rest and $\mu$ is the Possion's ratio. Bottom boundary is restricted from movement in any direction, while the two lateral boundaries are allowed to displace only in the vertical direction, in the fashion that the soil swelling within the surficial layer can be well modeled. The FEA model is enlarged in both horizontal and vertical directions in comparison to the original physical model to eliminate the effect of boundary conditions. It was found that 
the lower boundary selected for this study is deep enough, because the snow melting infiltration has a negligible effect on the flow regime near the lower boundary. The infinite elements are used for the two side boundaries. The infinite elements greatly extend the side boundary conditions far away from the main area of interest, which means snow melting infiltration has little effect on the hydraulic heads or suction profile far away in the horizontal direction, and vice versa. The use of infinite element will definitely increase the computational accuracy, especially for the flow behaviour in the region near the slope toe and slope shoulder.

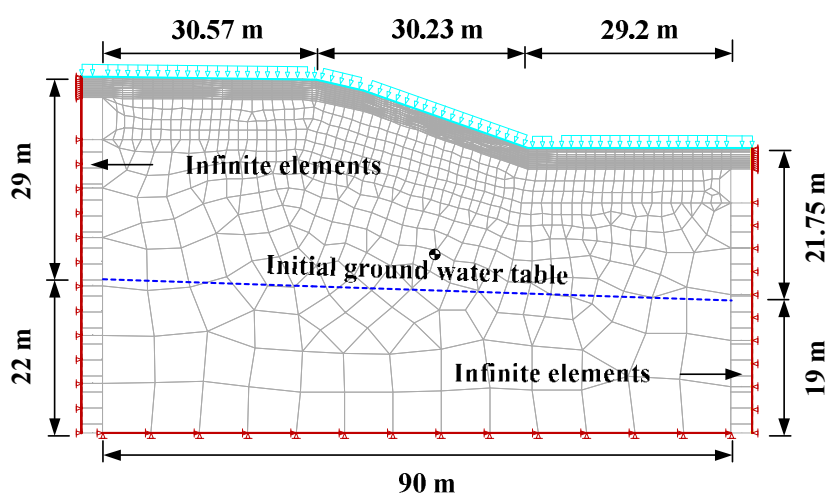

Fig. 3 Finite element method-based computational model

Different sizes of meshes were used for different zones within the computational model: finer quadrilateral element mesh for dealing with the dramatic response within the surficial layer where snow melting was predominant and larger size of mesh in other zones for ensuring the computational efficiency.

Widger and Fredlund (1978) illustrated that the matric suction of Regina clay at construction near optimum water content could range from 135 to $380 \mathrm{kPa}$ with a mean value of $270 \mathrm{kPa}$. The infiltration and evaporation during the period between the time of construction and failure may increase or decrease the suction value within the soil profile. However, an essential annual cyclic variation in the suction profile with the surficial layer can be expected. Such cyclic variations have been measured in other slopes, such as the field study conducted by Smethurst et al (2006) in a grass-covered cut slope in London Clay. In this study, the initial suctions within the surficial layer are assumed to be consistent with those at construction. This assumption is considered to be reasonable, because the environment is typically windy, and relative dry winter might bring the suction back to a higher value before snow melting in the spring season. The initial ground water table is constructed at the depth ranging from about 20 to $29 \mathrm{~m}$ under the slope surface as shown in Fig. 3. The hydrostatic variation of PWP along vertical distance with reference to the ground water table is formed to make the initial suction condition within the surficial range from around 200 to $300 \mathrm{kPa}$. Both the hydraulic heads at the right and left lateral boundaries increase linearly with depth below and above the ground water table, and remain consistent during snow melting. The bottom boundary of the computational domain for water flow has a zero flux. The infiltration from snow melting is simulated using an influx applied on slope ground surface with no ponding allowed.

\subsection{Soil properties}

Different soil properties of Regina clay were assigned to the two layers according to the physical model discussed in the previous section. Fig. 4 shows the SWCCs used in the coupled hydro-mechanical analysis. For the surficial layer, the bimodal SWCC measured by Azam and Ito (2011) is used to account for the effect of fissures and cracks on the water flow behaviour. Similar to a dual porosity structure soils (Li et al 2011, Li et al 2014), an increase in suction from the first (fissure) air-entry value (AEV) of $10 \mathrm{kPa}$ to the first residual value of $70 \mathrm{kPa}$ causes the desaturation primarily in the cracks (i.e. the macro-pores). The drainage of water in the intraaggregate pores (i.e. micro-pores) occurs when suction is beyond the second air-entry value of $300 \mathrm{kPa}$. For layer 2, the superposition technique (Fredlund et al 2012, Li et al 2011) is used here to derive the unimodal SWCC based on the bimodal SWCC measured by Azam and Ito (2011).

Fig. 5 illustrates the coefficient of permeability functions used in the present study. The unimodal permeability function assigned to layer 2 is from $\mathrm{Vu}$ and Fredlund (2004) who performed a numerical analysis of heave of slabon-grade floor on Regina clay induced by leakage of water line underground. This value of 


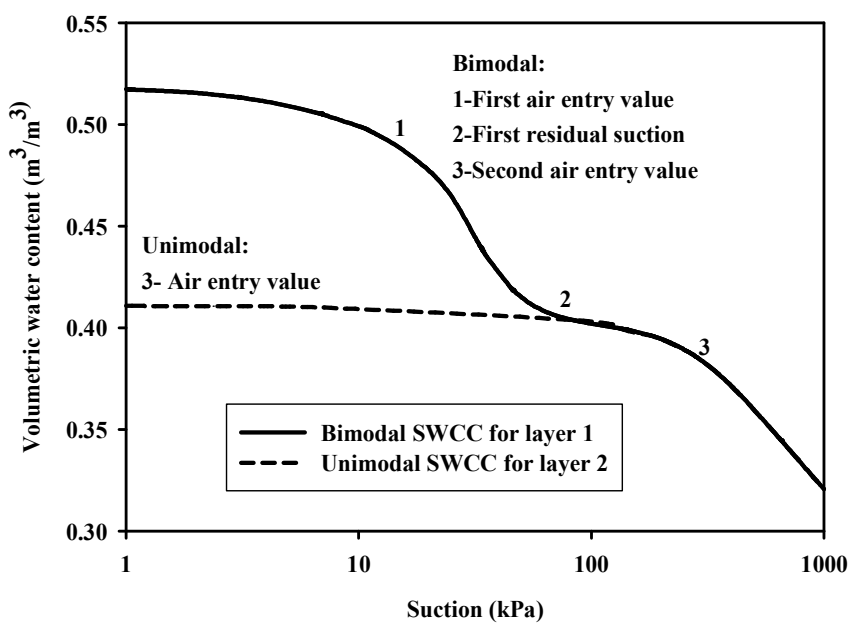

Fig. 4 SWCCs of Regina clay

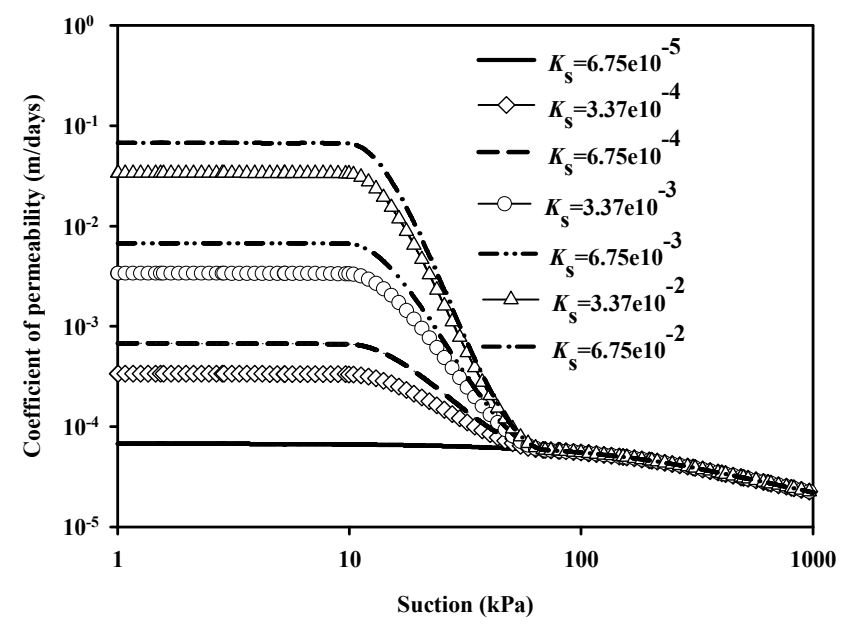

Fig. 5 Permeability functions of Regina clay

coefficient of permeability was originally measured by Shuai (1996) on compacted Regina clay specimen in the laboratory, with a saturated permeability of $6.75 \times 10^{-5} \mathrm{~m} /$ day. The presence of cracks and fissures can lead to an increase in the saturated coefficient of permeability by one or more magnitudes. The numerical analysis conducted on London Clay by Rouainia et al (2009) provided satisfactory results when the field saturated coefficient of permeability was increased by about 2 or 3 of magnitudes in comparison to the laboratory measured values. Limited information on the coefficient of permeability of cracked Regina clay is available in the literature. For this reason, a parametric analysis is undertaken in the present study to investigate the effect of the saturated coefficient of permeability on the FS. Six cases (i.e. assuming different saturated coefficient of permeability values for cracked soil increased by $0.5,1,1.5,2,2.5$, and 3 of magnitudes in comparison to laboratory measured value, respectively) are considered as shown in Fig. 5. For each case, a turning point occurs at the first residual suction on the permeability function to form the bimodal behavior which is consistent with the bimodal SWCC.

The mechanical properties used in the study are summarized in Table 1. Since the effect of cyclic environmental variation would lead to degradation in the shear strength parameters, the measured values on the sample at the time of failure by Widger and Fredlund (1978) was used for computing the FS. Furthermore, progressive failures were observed and reported by other researchers. For this reason, use of residual shear strength also deserves attention in the rigorous slope stability analysis. The finite element stress based slope stability analysis using both the peak and residual shear strength parameters was performed in this study.

Table 1 Soil mechanical properties from Widger and Fredlund (1978)

\begin{tabular}{l|cc}
\hline \multicolumn{1}{c|}{ Properties } & $\begin{array}{c}\text { Surface } \\
\text { layer }\end{array}$ & $\begin{array}{c}\text { Intact } \\
\text { soil }\end{array}$ \\
\hline Unit weight, $\gamma\left(\mathrm{kN} / \mathrm{m}^{3}\right)$ & 18 & 18 \\
\hline $\begin{array}{l}\text { Effective internal friction } \\
\text { angle, } \phi^{\prime}\left({ }^{\circ}\right)\end{array}$ & 20 & 17.5 \\
\hline $\begin{array}{l}\text { Effective cohesion, } \\
c^{\prime}(\mathrm{kPa})\end{array}$ & 0 & 39 \\
\hline $\begin{array}{l}\text { Residual effective internal } \\
\text { friction angle, } \phi_{r}{ }^{\prime}\left({ }^{\circ}\right)\end{array}$ & 13 & - \\
\hline $\begin{array}{l}\text { Residual effective } \\
\text { cohesion, } c_{r}{ }^{\prime}(\mathrm{kPa})\end{array}$ & 0 & - \\
\hline $\begin{array}{l}\text { Modulus of elasticity, } E \\
(\mathrm{MPa})\end{array}$ & $10^{*}$ & $10^{*}$ \\
\hline Poison's ration, $\mu$ & $0.33^{*}$ & $0.33^{*}$ \\
\hline
\end{tabular}

*Assumed values based on Adem and Vanapalli (2013) and Vu and Fredlund (2004) 


\section{Results and discussion}

\subsection{Variation of FS with time for peak shear strength}

In the spring, the snow on the ground in Regina gradually melts with increasing temperature and other environmental factors, which usually lasts for up to 2 months. In this study, an infiltration rate of $250 \mathrm{~mm} / \mathrm{month}$ (i.e. $8.3 \times 10^{-3} \mathrm{~m} /$ day) is applied to the slope surface to simulate the snow melting in Regina. During the 2 months of snow melting infiltration, the slope soil, especially within the surficial layer, will exhibit a significant volume increase, which would change the stress distribution within the slope profile. In the meantime, the suction of the soil would decrease with increasing water content. The stress change and suction decrease with the slope profile are captured using the hydro-mechanical model in the transient fashion with a time interval of one day. After each time interval, the output information from the hydro-mechanical model are input into the finite element stress based model to calculate the FS, In this way, the FS variation with time due to snow melting can be obtained.

Fig. 6 illustrates the variation of calculated FS with respect to time for different saturated coefficient of permeability values considered in this study. It can be seen from Fig. 6(a) that the FS decreases gradually and slowly with the snow melting when unimodal permeability function is used along with saturated coefficient of permeability which is equal to the laboratory measured value. For this case, FS is above 3.5 at the end of 2 months, which means the slope is still very stable and will not fail during snow melting. When the saturated coefficient of permeability is increased to $3.37 \times 10^{-4} \mathrm{~m} /$ day (increase by 0.5 of magnitude) or to $6.75 \times 10^{-4}$ $\mathrm{m}$ /day (increase by 1.0 of magnitude), the FS is still relatively high (greater than 2.5 for both cases), indicating a fairly stable slope. The variations in FS for cases where the saturated coefficient of permeability values are increased further are shown in Fig. 6(b). An increase in the saturated coefficient of permeability to $3.37 \times 10^{-3}$ $\mathrm{m}$ /day (increase by 1.5 of magnitude) substantially reduces the FS to about 1.2 at the end of snow melting. It can be expected that any other unfavorable factors, such as longer time period of snow melting, would cause the slope to fail for this case. When the saturated coefficient of permeability is increased to $6.75 \times 10^{-3} \mathrm{~m} /$ day (increase by 2 of magnitudes), $3.37 \times 10^{-2} \mathrm{~m} /$ day (increase by 2.5 of magnitudes), or $6.75 \times 10^{-2}$
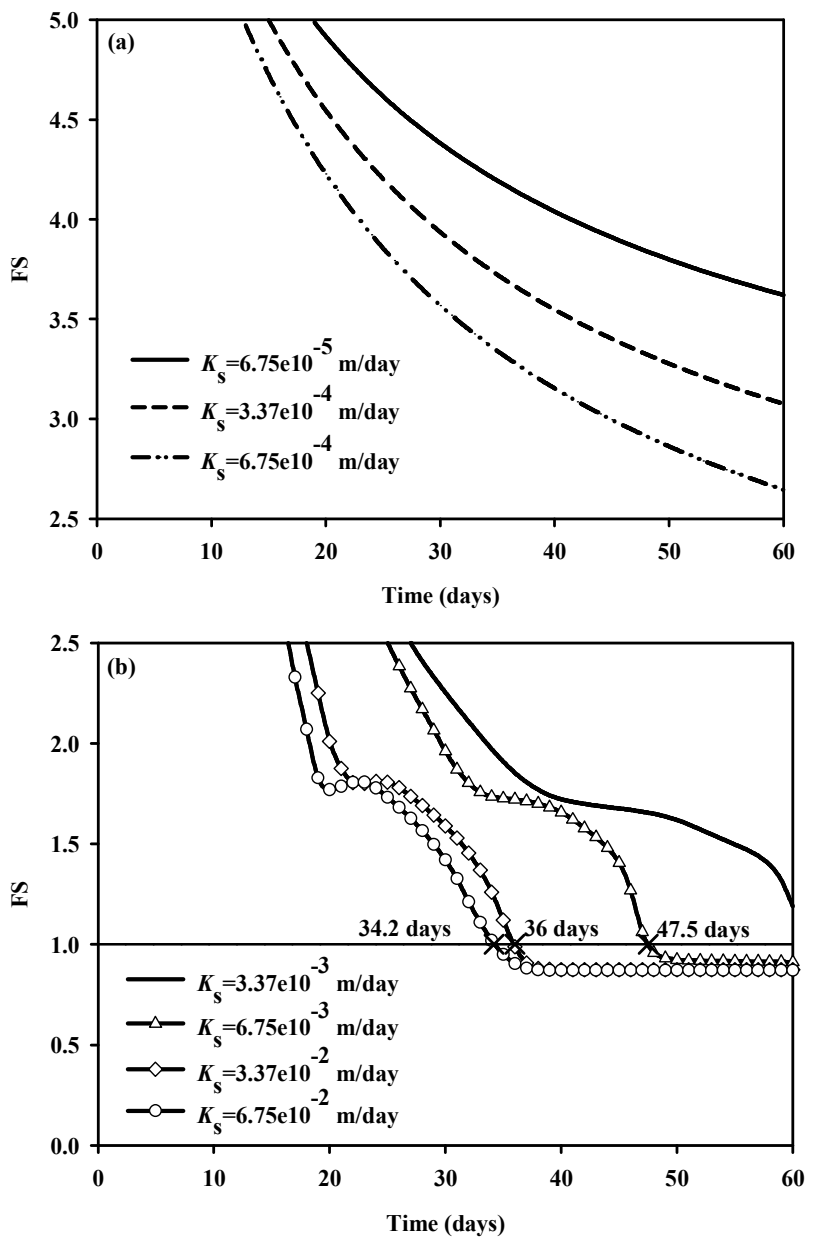

Fig. 6 Variation of FS for peak shear strength parameters: (a) $K_{\mathrm{S}}=6.75 \times 10^{-5}, 3.37 \times 10^{-4}, 6.75 \times 10^{-4} \mathrm{~m} /$ days; (b) $K_{\mathrm{S}}=$ $3.37 \times 10^{-3}, 6.75 \times 10^{-3}, 3.37 \times 10^{-2}, 6.75 \times 10^{-2} \mathrm{~m} /$ day

$\mathrm{m} /$ day (increase by 3 of magnitudes), a dramatic reduction in FS with time can be observed from Fig. 6(b). Slope failure (FS=1) occurs after 34.2, $36,47.5$ days of snow melting for all the three cases, respectively. The results illustrate that an increase in saturated coefficient of permeability in the bimodal permeability functions will shorten the time period for slope failure to occur during snow melting. The saturated coefficient of permeability is 2 to 3 of magnitudes higher than the measured value from laboratory tests due mainly to the cracks and fissures present in the surficial layer. It can be seen that there is 
insignificant difference in the time to failure when the saturated coefficient of permeability is increased from $3.37 \times 10^{-2} \mathrm{~m} /$ day to $6.75 \times 10^{-2}$ $\mathrm{m} /$ day. This is because both these values are greater than the boundary condition for snow melting $\left(8.83 \times 10^{-3} \mathrm{~m} /\right.$ day $)$ used in the present study. For this situation, the infiltration rate will be equal to the $8.83 \times 10^{-3} \mathrm{~m} /$ day and independent of the value of the saturated coefficient of permeability.

The effect of hydro-mechanical coupling on the FS is also investigated in this study. Fig. 7 provides FS variations comparisons based on coupled and uncoupled analysis for saturated coefficient permeability value equal to $6.75 \times 10^{-2}$ $\mathrm{m} /$ day. The uncoupled analysis herein means the volume change induced stress analysis is conducted after the completion of seepage analysis. For this reason, the effect of volume change of expansive soil on flow behavior is not considered. Fig. 7 shows that the uncoupled analysis can overestimate the FS as well as the time of failure significantly. This means use of uncoupled analysis in engineering practice may lead to a design on the unsafe side.

The underlying reason for the discrepancy between coupled and uncoupled analysis is further investigated by examining the PWP variation in both the analyses. Fig. 8 shows the variation of PWP with time at three points (Fig. 2) along the slip surface for coupled and uncoupled analysis. For all three points, the negative PWPs (i.e. suction value) for coupled analysis exhibit a

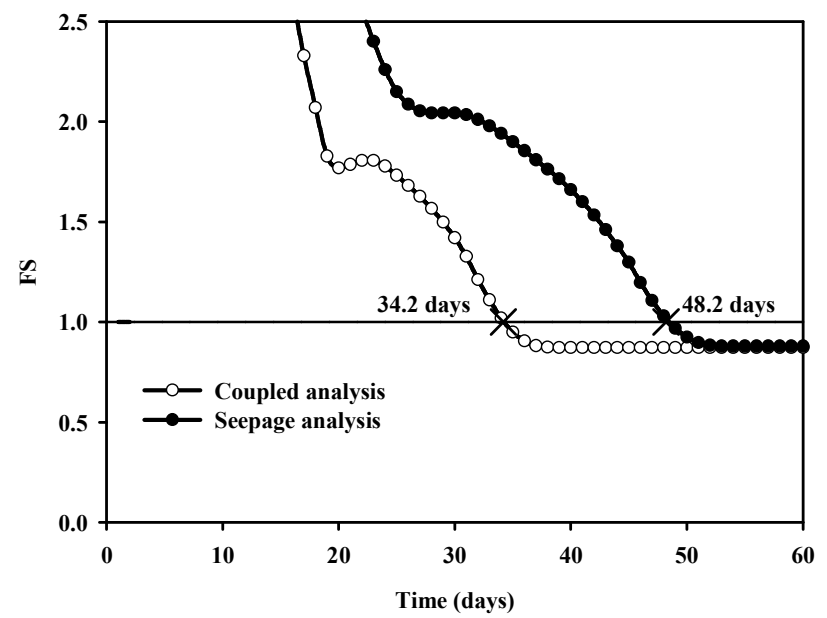

Fig. 7 Comparison in FS from coupled and uncoupled analysis for saturated permeability $=6.75 \times 10^{-2} \mathrm{~m} /$ day faster decrease than those resulting from the uncoupled analysis, which means, volume increase of expansive soil may speed the downward flow of water within the slope profile. A faster rate of suction loss will result in a faster shear strength reduction, contributing to early slope failure.
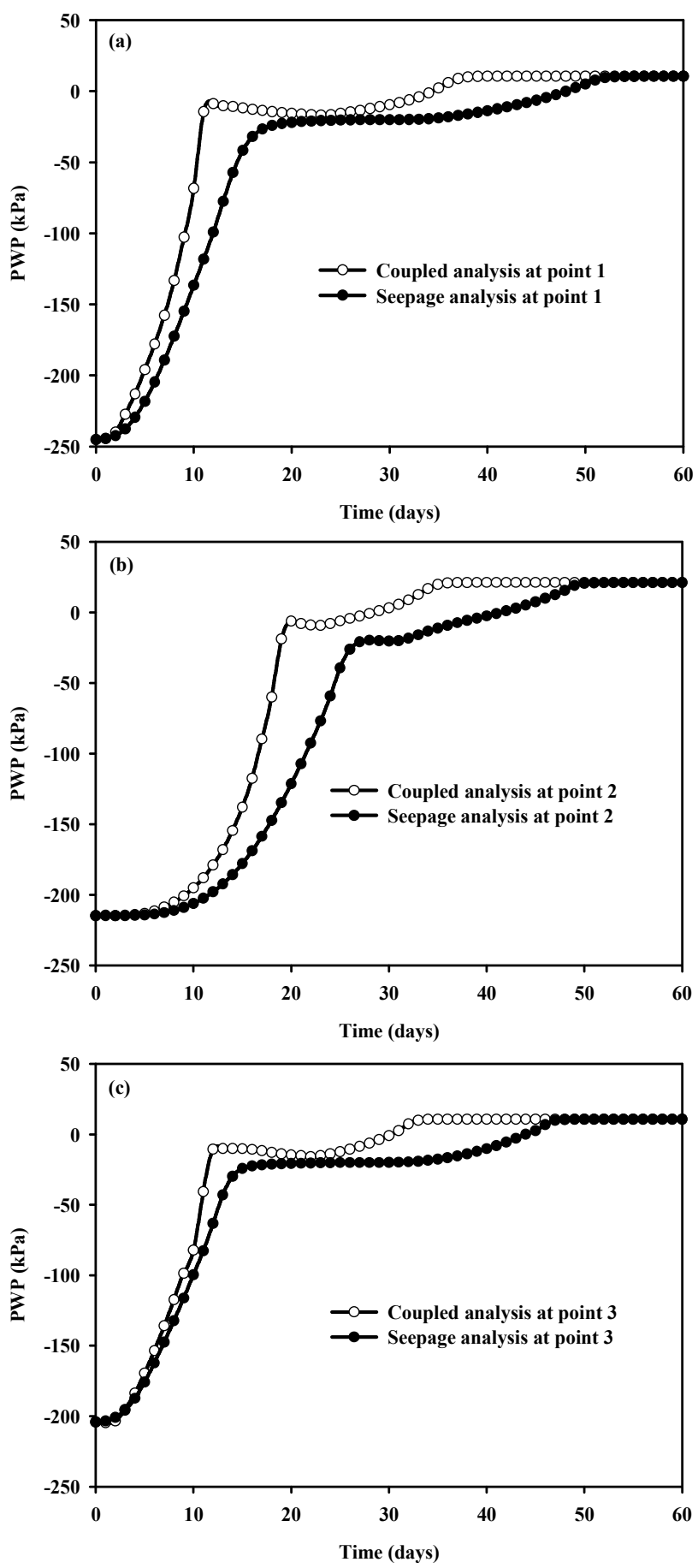

Fig. 8 Comparison in PWP from coupled and uncoupled analysis for $K_{\mathrm{S}}=6.75 \times 10^{-2} \mathrm{~m} /$ day: (a) at point 1 ; (b) at point 2 ; (c) at point 3 


\subsection{Variation of FS with time for residual shear strength}

Many studies showed the expansive soil slope failures exhibit a progressive failure. The triaxial as well as direct shear tests conducted by Widger and Fredlund (1978) illustrated strain-softening behavior for Regina clay. The numerical analysis conducted by Potts (1997) suggested that the average mobilized shear strength along the slip surface at the time of surface is between the peak and residual value. The residual shear strength parameters are also used in the present study, in order to provide a comprehensive picture about the instability of this slope under snow melting.

The FS variation based on the residual shear strength is shown in Fig. 9. It is clear that the trends in the FS variation are similar to those obtained using the peak shear strength parameters for all saturated coefficient of permeability values considered in the present study. However, the value of FS using the residual shear strength parameters is much lower. For example, when the saturated coefficient of permeability is increased to $6.75 \times 10^{-4} \mathrm{~m} /$ day (increase by 1.0 of magnitude), the FS is decreased to just above 1.5 (greater than 2.5 for peak shear strength parameters). However, the slope failure is still not triggered by the snow melting for saturated coefficient of permeability increase by 0.5 or 1 of magnitude.

When the saturated coefficient of permeability is increased by 1.5 to 3 of magnitudes, the FSs are all decreased to unity by snow melting during the 2 month period. Again, higher saturated coefficient of permeability in the bimodal permeability functions generally results in slope failure at an earlier time. However, when the saturated coefficient of permeability is increased beyond $3.37 \times 10^{-2} \mathrm{~m} /$ day, the time to failure will not significantly reduce anymore. Such a behavior can be attributed to the infiltration rate that is dependent on the flux boundary condition applied (e.g. snow melting rate) when the saturated coefficient of permeability is greater than the snow melting rate. For this reason, it can also be expected that a higher snow melting rate or a rain storm can lead to failure of this slope in a much shorter period of time when the saturated coefficient of permeability is increased by 2.5 or 3 of magnitudes.
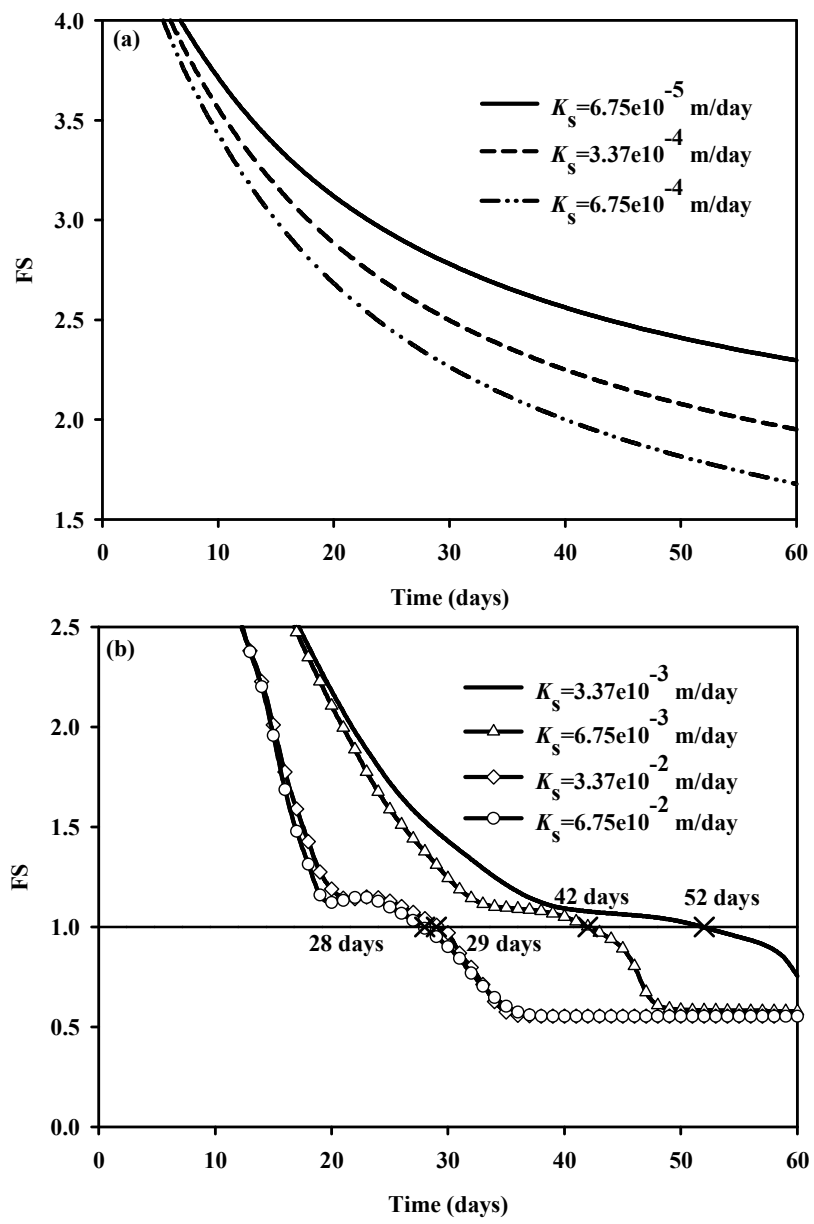

Fig. 9 Variation of FS for residual shear strength parameters: (a) $K_{\mathrm{S}}=6.75 \times 10^{-5}, 3.37 \times 10^{-4}, 6.75 \times 10^{-4} \mathrm{~m} /$ days; (b) $K_{\mathrm{S}}=3.37 \times 10^{-3}, 6.75 \times 10^{-3}, 3.37 \times 10^{-2}, 6.75 \times 10^{-2} \mathrm{~m} /$ day

\subsection{The failure mechanism and slip surface (or failure mode) of Regina clay slope}

Fig. 10 illustrates the flow regime within the surficial layer at the time of failure (i.e. when the FS is just below unity) for the fixed slip surface which was detected by Widger and Fredlund (1978). When using the peak shear strength parameters and $K_{\mathrm{S}}=6.75 \times 10^{-2} \mathrm{~m} /$ day, a perched water table forms within the cracked surficial layer at the time of failure. The perched ground water table (PWP is equal to zero) near the slope toe is at the ground surface, and the entire slip surface is almost below the ground water table except for a small part at the back of slip surface. This means the suction (negative PWP) that contributes the slope stability is almost completely lost due to snow melting, and the slope failure is caused by increasing PWP on the slip surface due to the formation of perched water 


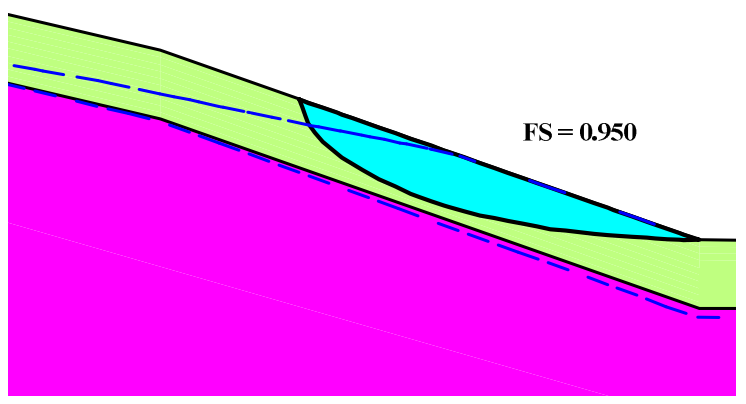

Fig. 10 Detected actual slip surface (peak shear strength parameters and $K_{\mathrm{S}}=6.75 \times 10^{-2} \mathrm{~m} /$ day after 34 days of snow melting)

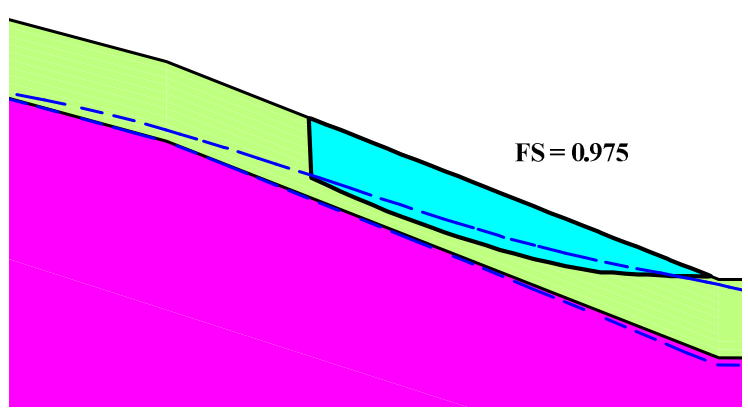

Fig. 11 Slip surface from an mathematical optimum algorithm (peak shear strength parameters and $K_{\mathrm{S}}=$ $6.75 \times 10^{-2} \mathrm{~m} /$ day after 31 days of snow melting)

table. The formation of perched water table essentially starts near the toe of slope, thus the slip surface intersects the slope toe. To validate the failure mechanism suggested in the present study, a mathematical optimum algorithm is used to determine the critical slip surface for the same case, which is shown in Fig. 11. The depth and shape of the mathematically optimized critical slip surface (Fig. 11) are essentially consistent with the actual failure surface (Fig. 10) observed and reported by Widger and Fredlund (1978). The formation of positive PWP at the slip surface is the main cause of slope failure. A small discrepancy in the FS value and the back of slip surface may be attributed to the heterogeneity of actual field condition and selected mathematical optimum algorithm. This may have led to a small difference in the predicted time of failure and corresponding water level.

\section{Conclusions}

The failure of a highway overpass embankment constructed of Regina clay at Belle Plaine is reanalyzed in this study using the state-of-the-art understanding of the mechanics of unsaturated soils. The shallow embankment failure is considered to be triggered by water infiltration of snow melting in the spring of 1973. A coupled hydro-mechanical finite element analysis is conducted to simulate infiltration-induced suction loss and swelling-induced stress change within the surficial layer. The FS variation with time during the period of snow melting is calculated using finite element stress based method using both the peak and residual shear strength parameters. The results show that the saturated coefficient of permeability measured from the laboratory tests on soil specimens is not representative of the actual field conditions. The effect of cracks and fissures within the surficial layer on the flow behavior as well as the slope stability can be reasonably well accounted by using the bimodal SWCC and bimodal permeability function with an increased saturated coefficient of permeability by 2-3 of magnitudes of measured value from laboratory tests. Use of residual shear strength parameters can also result in a much earlier slope failure during the period of snow melting and deserves more attention in conventional engineering practice for rational design of expansive soils slopes.

The flow regime at the time of failure predicted in this study are generally comparable with those simply assumed by Widger and Fredlund (1978) when using the limit equilibrium method for analysis of the same case. The gradual formation of perched water table causing the slope failure is successfully predicted using the coupled hydro-mechanical FEA. The importance of selecting proper coefficient of permeability is highlighted in this study. The approach presented in this study can provide a comprehensive analysis for practitioners regarding the slope stability of expansive soils taking account of the influence of environmental factors.

\section{Acknowledgements}

The first author gratefully acknowledges and appreciates the China Scholarship Council and the University of Ottawa, Canada for funding his PhD research program. The second author thanks the support from NSERC for his research programs. 


\section{References}

Adem, H.H. and S.K. Vanapalli, 2013. Constitutive modeling approach for estimating the 1-D heave with respect to time for expansive soils. International Journal of Geotechnical Engineering, 7(2): 199 - 204. DOI: 10.1179/1938636213Z.00000000024.

Alonso, E.E., A. Gens and C.H. Delahaye, 2003. Influence of rainfall on the deformation and stability of a slope in overconsolidated clays: a case study. Hydrogeology Journal, 11(1): 74 - 192. DOI: 10.1007/s10040-002-0245-1.

Aubeny, C.P. and R.L. Lytton, 2004. Shallow slides in compacted high plasticity clay slopes. Journal of Geotechnical and Geoenvironmental Engineering, 130(7): 717-727. DOI: $\quad 10.1061 /(\mathrm{ASCE}) 1090$ 0241(2004)130:7(717).

Azam, S. and M. Ito, 2011. Unsaturated soil properties of a fissured expansive clay. Proceedings, 64th Canadian Geotechnical Conference, Toronto, Canada. 313: 1 - 5.

Cheng, Y.H., Z.L. Cheng and Y.B. Zhang, 2011. Centrifugal model tests on expansive soil slope under rainfall. Chinese Journal of Geotechnical Engineering, 33(1): 409 - 414. (in Chinese)

Chowdhury, M.R.H., 2013. Shear strength properties of compacted expansive soils. Master's Thesis. Regina University, Canada.

Duncan, J.M. and S.G. Wright, 1995. Soil Strength and Slope Stability. Wiley, Hoboken, NJ, USA.

Flury, M., W. Fluhler, W.A. Jury and J. Leuenberger, 1994. Susceptibility of soils to preferential flow of water: a field study. Water Resource Research, 30(7): 1945 - 1954. DOI: 10.1029/94WR00871.

Fredlund, D.G. and N.R. Morgenstern, 1976. Constitutive relations for volume change in unsaturated soils. Canadian Geotechnical Journal, 13(3): 261 - 276. DOI: 10.1139/t76029.

Fredlund, D.G., H. Rahardjo and M.D. Fredlund, 2012. Unsaturated Soil Mechanics in Engineering Practice. John Wiley \& Sons, New York.

GeoSlope International Ltd., 2007a. Sigma/W User's Guide for Stress-Deformation Analysis.
GEO-SLOPE International Ltd, Calgary, Alta.

GeoSlope International Ltd., 2007b. Seep/W User's Guide for Finite Element Seepage Analysis. GEO-SLOPE International Ltd, Calgary, Alta.

GeoSlope International Ltd., 2007c. Slope/W User's Guide for Slope Stability Analysis. GEO-SLOPE International Ltd, Calgary, Alta.

Hamdhan, I.N. and H.F. Schweiger, 2013. Finite element method-based analysis of an unsaturated soil slope subjected to rainfall infiltration. International Journal of Geomechanics, 13(5): 653 - 658. DOI: 10.1061/ (ASCE) GM.1943-5622.0000239.

Hillel, D., 1998. Environmental soil physics. Academic Press, San Diego, California.

Ito, M. and S. Azam, 2009. Engineering characteristics of a glacio-lacustrine clay deposit in a semi-arid climate. Bulletin of Engineering Geology and the Environment, 68(4): 551 - 557. DOI: 10.1007/s10064-0090229-7.

Lam, L. and D.G. Fredlund, 1993. A general limit equilibrium model for three-dimensional slope analysis. Canadian Geotechnical Journal, 30(6): 905 - 919. DOI: 10.1139/t93-089.

Li, J.H., L.M. Zhang and X. Li, 2011. Soil-water characteristic curve and hydraulic conductivity function for unsaturated cracked soil. Canadian Geotechnical Journal, 48(7): 1010 - 1031. DOI: 10.1139/t11-027.

Li, X., J.H. Li and L.M. Zhang, 2014. Predicting bimodal soil-water characteristic curves and hydraulic conductivity functions using physically based parameters. Computers and Geotechnics, 57: 85 - 96. DOI: 10.1016/j.compgeo.2014.01.004.

Ling, D.S., S.C. Qi, F. Chen and N. Li, 2013. A limit equilibrium model based on Morgenstern-Price method for 3D slope analysis, Chinese Journal of Rock Mechanics and Engineering, 32(1): 107 - 116. (in Chinese)

Morgenstern, N.R. and V.E. Price, 1965. The analysis of the stability of general slip surfaces. Géotechnique, 15(1): 79 - 93. DOI: 10.1680/geot.1965.15.1.79.

Ng, C.W.W., L.T. Zhan, C.G. Bao, D.G. Fredlund and B.W. Gong, 2003. Performance 
of an unsaturated expansive soil slope subjected to artificial infiltration. Géotechnique, 53(2): 143-157.

DOI: 10.1680/geot.2003.53.2.143.

Pineda, J.A., E. Romero, M.D. Gracia and D. Sheng, 2014. Shear strength degradation in claystones due to environmental effects. Géotechnique, 64(6): 493-501. DOI: 10.1680/geot.13.T.025.

Potts, D.M., N. Kovacevic and P.R. Vaughan, 1997. Delayed collapse of cut slopes in stiff clay. Géotechnique, 47(5): 953 - 982. DOI: 10.1680/geot.1997.47.5.953.

Rahardjo, H., T.T. Lim, M.F. Chang and D.G. Fredlund, 1995. Shear strength characteristics of a residual soil. Canadian Geotechnical Journal, 32(1): 60 - 77. DOI: 10.1139/t95-005. Richards, L.A., 1931. Capillary conduction of liquids through porous mediums. Physics, 1(5): 318 - 33. DOI: 10.1063/1.1745010.

Rouainia, M.O., T.O. Davies and S. Glendinning, 2009. Numerical modelling of climate effects on slope stability. Proceedings of the Institution of Civil Engineers Engineering Sustainability, 162(ES2): 81 - 89. DOI: 10.1680/ensu.2009.162.2.81.

Shuai, F., 1996. Simulation of swelling pressure measurements on expansive soils. Ph.D. Thesis, University of Saskatchewan, Saskatoon, SK, Canada.

Smethurst, J.A., D. Clarke and W. Powrie, 2006. Seasonal changes in pore water pressure in a grass-covered cut slope in London Clay. Géotechnique, 56(8): 523 - 537. DOI: 10.1680/geot.2006.56.8.523.
Vanapalli, S.K., D.G. Fredlund, D.E. Pufahl and A.W. Clifton, 1996. Model for the prediction of shear strength with respect to soil suction. Canadian Geotechnical Journal, 33(3): 379 392. DOI: 10.1139/t96-060.

Vu, H.Q. and D.G. Fredlund, 2004. The prediction of one-, two-, and three dimensional heave in expansive soils. Canadian Geotechnical Journal, 41(10): 713 737. DOI: 10.1139/T04-023.

Widger, R.A. and D.G. Fredlund, 1978. Stability of swelling clay embankments. Canadian Geotechnical Journal, 16(1): 140 - 151. DOI: 10.1139/t79-012.

Wong, T.T., D.G. Fredlund and J. Krahn, 1998. A numerical study of coupled consolidation in unsaturated soils. Canadian Geotechnical Journal, 35(6): 926 - 937. DOI: 10.1139/t98065.

Xu, G.M., G.L. Wang, X.W. Gu and Y.J. Zeng, 2006. Centrifuge modeling for instability of excavated slope in expansive soil due to water infiltration. Chinese Journal of Geotechnical Engineering, 28(2): 270 - 273. (in Chinese)

Yoshida, R.T., D.G. Fredlund and J.J. Hamilton, 1983. The prediction of total heave of a slabon-grade floor on Regina clay. Canadian Geotechnical Journal, 20(1): 69 - 81. DOI: 10.1139/t83-008.

Zhan, L.T., C.W.W. Ng and D.G. Fredlund, 2007. Field study of rainfall infiltration into a grassed unsaturated expansive soil slope. Canadian Geotechnical Journal, 44(4): 392 408. DOI: 10.1139/t07-001. 